\title{
Evaluation of biochemical and clinical markers of endothelial dysfunction and their correlation with urinary albumin excretion in patients with type 1 diabetes mellitus
}

Sefika Burcak Polat', Nagihan Ugurlu², Nabi Aslan³, Neslihan Cuhaci', Reyhan Ersoy', Bekir Cakir ${ }^{1}$

\begin{abstract}
Objective: Endothelial dysfunction (ED) plays an important role in the pathogenesis of diabetic nephropathy. The purpose of the study was to determine flow mediated endothelial dependent vasodilatation (FMD) measurements and serum soluble (s) endothelin-1 (ET-1), intercellular adhesion molecule-1 (ICAM-1) and vascular cell adhesion molecule (VCAM-1) levels in patients with type 1 diabetes mellitus (T1DM) with or without increased urinary albumin excretion (UAE) and compare them with the healthy controls. Subjects and methods: Seventy three patients with T1DM were enrolled. Patients were divided into two subgroups according to microalbumin measurements in 24-hr urine collections. The diabetic patients without microalbuminuria (41 patients) were defined as Group I and those with microalbuminuria (32 patients) were defined as group II. A hundred age and sex matched healthy subjects participated as the control group (Group III). Serum sET-1, sICAM-1, sVCAM-1 levels and FMD measurements were determined in all participants. Results: Median FMD measurement was significantly lower in the diabetic groups compared with the control group $(6.6,6.4$ and $7.8 \%$ in Group I, II and III, respectively) ( $p<0.05)$. FMD was negatively correlated with age $(p=0.042)$. Median serum sICAM-1 level was higher in the patient groups compared to the control group $(p<0.05)$. Median serum sVCAM- 1 level was higher in the group of patients with increased albuminuria compared to the normoalbuinuric and the control group $(p<0.05)$. Serum sVCAM-1 level was found to be positively correlated with degree of urinary albumin excretion $(p<0.001)$. Conclusion: We assume that sVCAM-1 may be used as a predictive marker for risk stratification for nephropathy development and progression. Arch Endocrinol Metab. 2016;60(2):117-24
\end{abstract}

Keywords

Nephropathy; ET-1; VCAM-1; ICAM-1; FMD; endothelial dysfunction
${ }^{1}$ Yildirim Beyazit University, Ataturk Education and Research Hospital, Department of Endocrinology and Metabolism, Ankara, Turkey ${ }^{2}$ Yildirim Beyazit University, Ataturk Education and Research Hospital, Department of Ophtalmology, Ankara, Turkey ${ }^{3}$ Yildirim Beyazit University, Ataturk Education and Research Hospital, Department of Cardiology, Ankara, Turkey

Correspondence to: Sefika Burcak Polat

Yildirim Beyazit University, Ataturk Education and Research Hospital, Department of Endocrinology and Metabolism 06800 - Bilkent, Ankara burcakugurlu@gmail.com

Received on Apr/28/2015 Accepted on Aug/28/2015

DOI: 10.1590/2359-3997000000116

\section{INTRODUCTION}

$\mathrm{T}$ ype 1 diabetes mellitus (TIDM) is a chronic metabolic disease characterized with autoimmune pancreatic beta cell destruction affecting young adults. During the course of the disease micro- and macrovascular complications develop. Diabetic nephropathy (DN) occurs in $30-40 \%$ of the patients and is the major cause of end stage renal disease (ESRD) and one of the major causes of morbidity and mortality in TIDM (1). Once ESRD develops, costly therapies such as hemodialysis or transplantation are required. Overt nephropathy is usually preceded by increased albuminuria which is not only associated with the risk of developing renal insufficiency but also with the development and progression of cardiovascular disease (2). Thus, there is considerable interest in determining the mechanisms responsible for albuminuria and in identifying early markers that may be associated with that complication of diabetes.

Well known clinical risk factors for progression of nephropathy are increased urinary albumin excretion (UAE), hypertension (HT), and hyperglycemia (3). Although strict glycemic control and the regulation of blood pressure reduce microvascular complications, nephropathy can still progress in some patients suggesting presence of other pathophysiologic mechanisms.

Inflammation and endothelial dysfunction (ED) were hypothesized to play an active role in the pathogenesis of DN (4). The term ED refers to an impairment of the ability of the endothelium to properly maintain vascular homeostasis (5). Although the term is often used in reference to a loss of bioavailable nitric oxide $(\mathrm{NO}), \mathrm{ED}$ also reflects increased production of vaso- 
constrictors and disturbed regulation of inflammation, thrombosis, and cell growth in the vascular wall $(5,6)$.

In the early phase of ED, an imbalance between endothelium-derived vasodilator and vasoconstrictor substances develops (7). Endothelin-1 (ET-1) is not only one of the most potent vasoconstrictors but it also has pro-inflammatory and profibrotic effects, which may contribute to the pathogenesis of nephropathy. In the previous studies, it was demonstrated that serum ETl level was increased in diabetic patients with microalbuminuria, retinopathy or higher glycosylated hemoglobin (HbAlc) (8-10).

In a normal endothelium NO creates an anti-inflammatory environment and prevents adhesion molecules to bind the inner surface of the vessels. However in presence of DM, NO is reduced, expression of vascular and intercellular adhesion molecules is increased allowing leucocytes to attach endothelial surface easily and progression of inflammation (11). Expression of those molecules was shown to be increased in retina, arterial walls and glomerulus of the diabetic patients (12). Previous studies reported a positive correlation between those molecules and rate of the complications (13). Intercellular adhesion molecule-1 (ICAM-1) and vascular cell adhesion molecule (VCAM-1) were shown to increase leucocyte adhesion on retinal vessels beginning from the earliest stages of diabetic retinopathy (DR) and correlates with presence of gross proteinuria (14). In another study, in the rat model of streptozotocininduced diabetes, retinal leukostasis increased within days of developing diabetes and correlated with the increased expression ICAM-1 and its blockade with a specific antibody prevented diabetic retinal leukostasis and vascular leakage by $48.5 \%$ and $85.6 \%$, respectively (15).

Flow mediated endothelial dependent vasodilatation (FMD) is one of the most extensively used non-invasive technique for assessment of endothelial function. It determines the degree of endothelial dysfunction on the basis of NO production capacity of the brachial artery. There are reports in the literature showing decreased FMD in patients with diabetic nephropathy (16).

The aims of the present study are: 1) to determine serum sICAM-1, VCAM-1, ET-1 levels and FMD measurements in TIDM patients with or without increased UAE and compare them with the control group; 2) to search the correlation between the UAE and those biochemical markers of ED in diabetic patients; 3 ) to determine the correlation between biochemical markers and a clinical marker (FMD) of ED.

\section{SUBJECTS AND METHODS}

\section{Study design}

Seventy-three patients with TIDM (36 female and 37 male) who regularly visited the Endocrinology Clinics between December 2011 and December 2012 and a hundred age and gender matched healthy individuals were enrolled in the present study. Each patient accepted and signed informed consent forms. The local ethics committee approved the study protocol.

Patient selection was made by the same endocrinologist on the basis of the following inclusion criteria: TIDM (as defined by deficient C-peptide secretion and autoantibody positivity at the time of diagnosis), duration of disease longer than 5 years. Patients with smoking history, coronary heart disease, congestive heart failure, peripheral artery disease, renal failure or chronic liver disease were excluded from the study.

Seventy-three patients with TIDM divided in two subgroups. Group I was defined as those with normal UAE (4l patients), and Group II was defined as those with increased UAE ( 32 patients). Microalbumin excretion more than $30 \mathrm{mg} / 24 \mathrm{hr}$ was defined as increased UAE. A hundred healthy, nondiabetic subjects (50 male, 50 female) were recruited from hospital staff to serve as the control group (Group III).

After detailed medical history (demographic data, duration of diabetes, date of initial diagnosis, smoking history, comorbidities, recent medications) was recorded, physical examination was performed for each participant. Systolic and diastolic blood pressures (SBP)/(DBP) were measured twice in the seated position after at least 15 minutes of resting. HT was defined as SBP $\geq 140$ $\mathrm{mmHg}, \mathrm{DBP} \geq 90 \mathrm{mmHg}$ or the current use of antihypertensive medications. All subjects underwent detailed ophthalmologic examination, including best-corrected visual acuity, applanation tonometry, anterior segment slit lamp biomicroscopy, and dilated fundus examination for presence of retinopathy and the data was recorded.

\section{Laboratory examination}

Blood samples were drawn into plain vacutainers from the antecubital veins of healthy controls and patients after $12 \mathrm{hr}$ fast and $30 \mathrm{~min}$ of supine rest between 8 and 9 a.m. Blood samples were sentrifuged at 4000 rpm for 10 minutes and serum was transferred in the eppendorf tubes. All serum samples were kept in $-80^{\circ} \mathrm{C}$ freezer and were run at the same time for biochemical evaluation. sET-1, sICAM-1 and sVCAM-1 levels were 
studied with commercial elisa kits (sET-1; Biomedica Gruppe, sICAM-1 and sVCAMI; Bender medical systems GmbH Viena, Austria). All intra- and interassay coefficient of variation $(\mathrm{cv})$ was 4.0/6.0, 4.1/4.7 and 3.1/5.2 for ET-1, ICAM-1 and VCAM-1, respectively.

Screening for increased urinary albumin excretion (> $30 \mathrm{mg} /$ day, Roche Diagnostics, Manheim, Germany) was performed by measurement of the albumin in 24-h or timed urine collections.

Glycated hemoglobin (HbAlc) was measured by high-performance liquid chromatography (HPLC), and the nondiabetic range used was 4.0-6.0\% (30-60 $\mathrm{mmol} / \mathrm{mol}$ ). To measure serum glucose and total cholesterol, triglycerides (TG), high density lipoprotein cholesterol (HDL) and low density lipoprotein cholesterol (LDL) measurements were made by an autoanalyzer using enzymatic methods (Roche Diagnostics ${ }^{\circledR}$ ).

\section{FMD measurements}

All measurements were taken in the morning, in a quiet, air-conditioned room maintained at $23^{\circ} \mathrm{C}$. The subjects rested quietly in the supine position for 20 min before and during each FMD measurement. Just before the cuff inflation, systolic and diastolic blood pressure was measured on the left arm. Brachial artery FMD was used as a measure of endothelium dependent vasodilation (EDV) using techniques previously described $(17,18)$. In the supine state, ultrasound imaging $(7 \mathrm{MHz}$, Acuson $128 \mathrm{XP}$, Mountain view, CA) of the brachial artery was performed in a longitudinal plane at a site $1-3 \mathrm{~cm}$ proximal to the antecubital fossa of the arm. Baseline images were recorded and a blood pressure cuff was placed on the forearm and inflated to $50 \mathrm{mmHg}$ above SBP for 5 min. Arterial diameter was determined during peak hyperemia after release of the blood pressure cuff from the forearm. To assess dilation, $10 \mathrm{~s}$ of images were captured 1-3 min after cuff release. Flow velocity was recorded at baseline and just after cuff release where maximal velocity was observed. FMD was defined as the ratio of flow mediated dilation to the basal measurement of the arterial diameter (maximum diameterbasal diameter/basal diameter x100).

\section{Statistical analysis}

All analyses were performed using the SPSS for Windows software package (ver. 11.5, IBM Corp, Armonk, NY, USA). The nonparametric Kolmogorov-Smirnov test was used to compare samples with the reference probability distribution, whereas the homogeneity of the variances was assessed using Levene's test. Descriptive statistics are presented as means $\pm \mathrm{SD}$, with medians (minimum-maximum) for continuous variables and percentages (\%) for categorical variables. The significance of the differences between mean values was assessed by oneway ANOVA. To assess significance of difference between median values, Kruskal-Wallis test was used when there were more than two groups to compare whereas Mann Whitney U test was used when there were only two groups to compare. If significant differences were detected after one-way ANOVA or Kruskal-Wallis tests, post hoc Tukey's HSD or Conover's nonparametric multivariance comparison tests were used to define the effective factors. For categorical variables, differences were assessed by chi squared and Fisher's exact tests, as appropriate. A value of $\mathrm{p}<0.05$ was considered to indicate statistical significance.

\section{RESULTS}

Seventy three patients with TIDM and a hundred healthy controls were enrolled in the study. Forty one patients $(56.1 \%)$ with TIDM had normal UAE (Group I) while thirty two (43.9\%) had increased UAE (Group II). Demographic data of the patient (Group I and II) and control group (Group III) was listed on Table 1. Sex distribution and mean age was similar between the groups. Median DM duration was 8 (5-40) years in Group I and 11.5 (5-35) years in Group II. HT was present in 4 patients $(9.8 \%)$ in Group I, 7 patients $(22.6 \%)$ in Group II and 4 patients $(4 \%)$ in group III (control group, $\mathrm{n}=100)(\mathrm{p}=0.087)$ (Table 1$)$. Mean serum LDL and median TG were similar between groups. The mean HDL was significantly lower in Group I compared to Group III (56 vs $47 \mathrm{mg} / \mathrm{dL}$, $\mathrm{p}<0.01)$. The median serum HbAlc levels were similar in the diabetic groups (group I and II) and were increased significantly, compared with the control group, as expected $(\mathrm{p}<0.001$; Table 1$)$.

Fundoscopic examination of the diabetic patients revealed that only 9 of 41 patients (28\%) in Group I had DR ( 8 patients had non proliferative diabetic retinopathy (NPDR) and 1 had proliferative diabetic retinopathy (PDR). In Group II fundoscopic examination of 3 patients $(9.4 \%)$ was normal, $15(46.9 \%)$ had NPDR and $14(43.8 \%)$ had PDR. Prevalence of DR was significantly higher in Group II compared to Group I $(\mathrm{p}<0.05)$. 
Median sET-1 level was $8.1,7.7,10.5 \mathrm{fmol} / \mathrm{l}$ in Group I, Group II and Group III, respectively and didn't differ significantly between the groups $(\mathrm{p}=$ 0.066) (Table 2, Figure 1A). Median sICAM-1 level was significantly higher in diabetic groups (Group I and II) compared to the control group (Group III) (Group I vs Group III; p < 0.001, Group II vs Group III; p = 0.05 ) whereas it was similar between Group I and II (Table 2, Figure 1B).

Median sVCAM-1 level was significantly higher in Group II compared to Group I and III (Group II $v s$ Group I; $\mathrm{p}<0.001$, Group II vs Group III; $\mathrm{p}<0.05$ ). There wasn't any significant difference between Group I and III (Table 2, Figure 1C).

Median FMD measurement was 6.6, 6.4 and 7.8\% in Group I, II and III, respectively. It was significantly lower in Group I and II compared to group III whereas there was no difference between Group I and group II (Group I vs Group III; $\mathrm{p}=0.002$, Group II vs Group III; p = 0.039) (Table 3, Figure ID).
Any significant correlation wasn't detected between FMD, ICAM-1, VCAM-l, ETl and HbAlc (p > 0.05) in diabetic patients. FMD was negatively correlated with age $(\mathrm{p}=0.042)$ (Table 4$)$. FMD was not correlated with duration of diabetes (Table 4 ).

A positive correlation was detected between serum VCAM- 1 level and degree of urinary albumin excretion $(\mathrm{p}<0.001)$ whereas serum ICAM-1 and ETl were not correlated with the degree of UAE (Table 4).

Table 3. Comparison of median FMD measurements between the groups

\begin{tabular}{|c|c|}
\hline Groups & FMD (\%) \\
\hline Group I $(n=41)$ & $6.6(3.1-10.3)^{\mathrm{a}}$ \\
\hline Group II ( $n=32)$ & $6.4(4.3-11.1)^{b}$ \\
\hline Group III $(n=100)$ & $7.8(3.1-12.0)^{a, b}$ \\
\hline$p$-value ${ }^{\dagger}$ & 0.013 \\
\hline
\end{tabular}

FMD: flow mediated endothelial dependent vasodilatation. Group I: T1DM patients without microalbuminuria, Group II: T1DM patients with microalbuminuria, Group III: Control Group. a: Difference between Group III and Group I is significant $(p=0.002)$, b: Difference between Group III and Group II is significant $(p=0.039),{ }^{\dagger}$ Kruskal Wallis test.

Table 1. Demographic, clinical and biochemical data of the groups

\begin{tabular}{|c|c|c|c|c|}
\hline Variables & Group I $(n=41)$ & Group II ( $n=32)$ & Group III $(n=100)$ & $p$-value \\
\hline Age (years) & $33.3 \pm 9.0$ & $33.7 \pm 10.7$ & $35.7 \pm 7.7$ & $0.060^{+}$ \\
\hline Female/male & $20 / 21$ & $16 / 16$ & $50 / 50$ & $0.992^{\ddagger}$ \\
\hline DM duration & $8(5-15)$ & $11.5(5-25)$ & - & 0.059 \\
\hline HT & $4(9.8 \%)$ & $7(22.6 \%)$ & $1(4.0 \%)$ & $0.087^{\#}$ \\
\hline LDL mg/dL & $99.0 \pm 39.2$ & $115.2 \pm 37.7$ & $113.3 \pm 3.6$ & $0.139^{+}$ \\
\hline $\mathrm{HDL} \mathrm{mg} / \mathrm{dL}$ & $47(31-68)^{a}$ & $50(27-110)$ & $56(29-88)^{a}$ & $0.016^{\$}$ \\
\hline $\mathrm{TG} \mathrm{mg} / \mathrm{dL}$ & $93(45-220)$ & $100(47-333)$ & $109(46-513)$ & $0.440^{\$}$ \\
\hline Creatine mg/dL & $0.89(0.50-1.60)$ & $0.80(0.60-2.10)$ & $0.70(0.4-1.2)$ & $0.680^{\$}$ \\
\hline $\mathrm{HbA1c} \%$ & $8.9(5.6-12.0)^{\mathrm{a}}$ & $9.2(6.0-12.3)^{b}$ & $5.3(4.4-6.3)^{a, b}$ & $<0.001^{\$}$ \\
\hline
\end{tabular}

Group I: T1DM patients without microalbuminuria, Group II: T1DM patients with microalbuminuria, Group III: Control Group. a: difference between Group III and Group I is significant ( $p<0.01$ ), b: difference between Group II and Group III is significant $(p<0.001) .{ }^{\dagger}$ One-Way ANOVA, ${ }^{\ddagger}$ Pearson'un chi-Square testi, " Mann Whitney U testi, " likelihood ratio test, ${ }^{\$}$ Kruskal Wallis test.

Table 2. Comparison of ET-1, ICAM-1 and VCAM-1 levels between the groups

\begin{tabular}{lccc}
\hline Groups & $\begin{array}{c}\text { sET-1 Median } \\
\text { (minimum maximum) (fmol/l) }\end{array}$ & $\begin{array}{c}\text { sICAM-1 Median } \\
\text { (minimum-maximum) (ng/mL) }\end{array}$ & $\begin{array}{c}\text { sVCAM-1 Median } \\
\text { (minimum maximum) (ng/mL) }\end{array}$ \\
\hline Group I $(\mathrm{n}=41)$ & $8.1(0.6-16.5)$ & $5100(1450-12860)^{\mathrm{a}}$ & $736.5(419.9-1516.7)^{\mathrm{c}}$ \\
Group II $(\mathrm{n}=32)$ & $7.7(3.1-23.5)$ & $4520(326.1-11070)^{\mathrm{b}}$ & $975.9(352.2-1525.5)^{\mathrm{b}, \mathrm{c}}$ \\
Group III $(\mathrm{n}=100)$ & $10.5(3.0-19.0)$ & $685.7(120-14460)^{\mathrm{a}, \mathrm{b}}$ & $680.1(380.0-1041.9)^{\mathrm{b}}$ \\
p-value $^{\dagger}$ & 0.066 & $\mathbf{< 0 . 0 0 1}$ & $\mathbf{0 . 0 0 2}$ \\
\hline
\end{tabular}

ET-1: endothelin-1, ICAM-1: intercellular adhesion molecule-1, VCAM-1: vascular cell adhesion molecule, Group I: T1DM patients without microalbuminuria, Group II: T1DM patients with microalbuminuria, Group III: Control Group. a: difference between group I and III is significant $(p<0.001)$, b: difference between Group II and III is significant $(p<0.05)$, c: difference between Group I and II is significant $(\mathrm{p}<0.001),{ }^{\dagger}$ Kruskal Wallis test. 

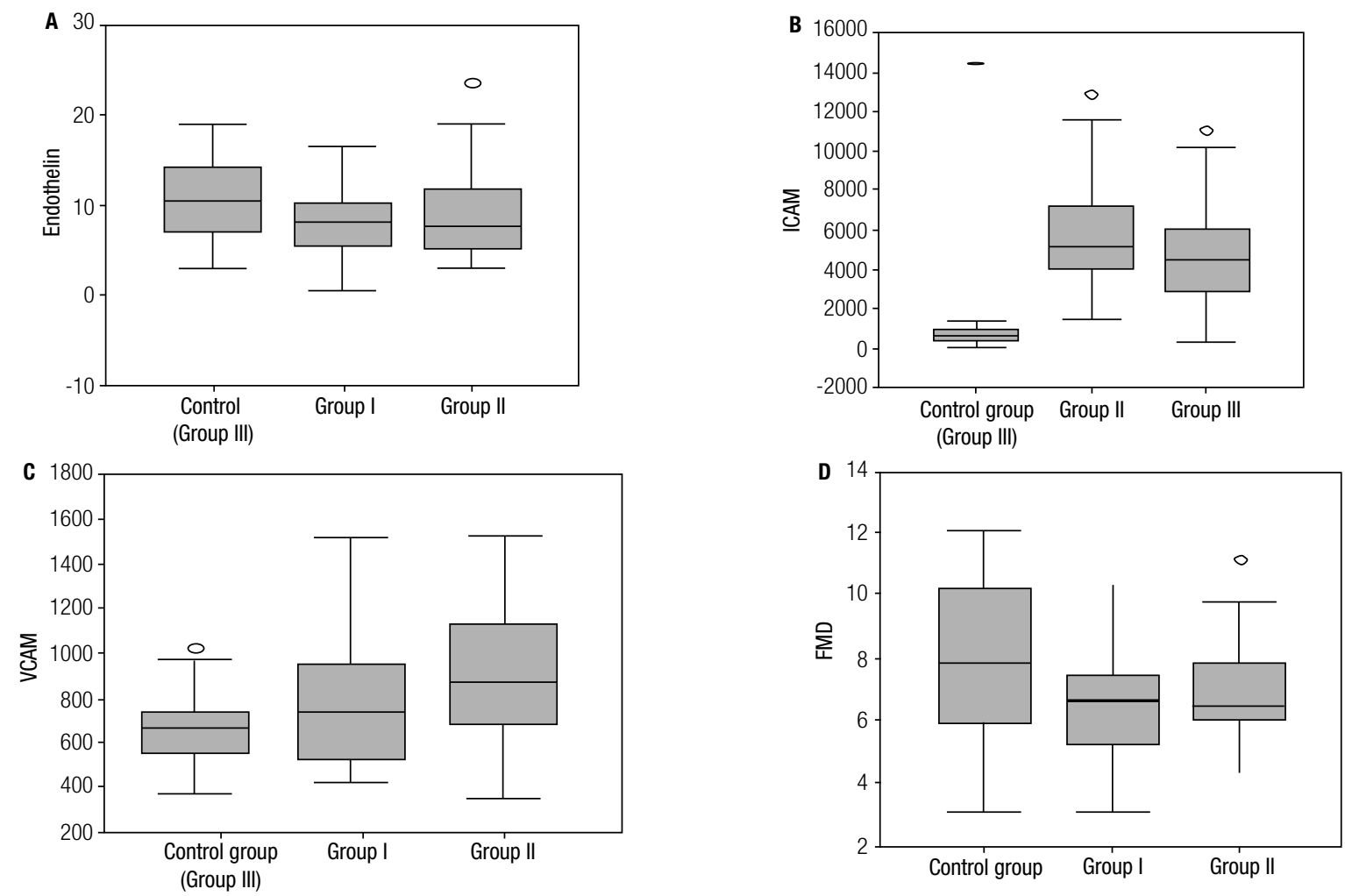

Figure 1. Distribution of ET-1, ICAM, VCAM and FMD in Group I, II and III is depicted in Figure A, B, C and D.

ET-1: endothelin-1, ICAM-1: intercellular adhesion molecule-1, VCAM-1: vascular cell adhesion molecule,

Group I: T1DM patients without microalbuminuria, Group II: T1DM patients with microalbuminuria, Group III: Control Group, Kruskal Wallis test was used and $p$ value was $0.06,<0.01,0.02$ and 0.013 for ET1, ICAM-1, VCAM-1 and FMD, respectively.

Table 4. Correlation of biochemical and clinical markers of endothelial dysfunction with certain parameters in diabetic patients

\begin{tabular}{lccccccccc}
\hline Variables & \multicolumn{2}{c}{ ICAM-1 } & \multicolumn{2}{c}{ VCAM-1 } & \multicolumn{2}{c}{ ET-1 } & & FMD \\
\hline & r-value & $p$-value & $r$-value & $p$-value & $r$-value & $p$-value & r-value & $p$-value ${ }^{\dagger}$ \\
Age & 0.038 & 0.755 & 0.044 & 0.724 & -0.063 & 0.608 & -0.247 & $\mathbf{0 . 0 4 2}$ \\
DM duration & -0.151 & 0.202 & 0.100 & 0.401 & 0.110 & 0.352 & 0.002 & 0.985 \\
HbA1c & -0.043 & 0.725 & -0.001 & 0.994 & 0.144 & 0.242 & 0.209 & 0.087 \\
UAE & -0.085 & 0.472 & 0.443 & $<0.001$ & -0.084 & 0.480 & -0.016 & 0.891 \\
\hline
\end{tabular}

ET-1: endothelin-1, ICAM-1: intercellular adhesion molecule-1, VCAM-1: vascular cell adhesion molecule, FMD: flow mediated endothelial dependent vasodilatation, HDA1c: glycosylated hemoglobin, UAE: urinary albumin excretion, ${ }^{\dagger}$ Spearman correlation test.

\section{DISCUSSION}

Endothelial dysfunction is a broad term which implies dysregulation of endothelial cell functions, including impairment of the barrier functions of endothelial cells, vasodilation, disturbances in proliferative capacities, migratory as well as tube formation properties, angiogenic properties, attenuation of synthetic function, and deterrence of white blood cells from adhesion and diapedesis (19). In the previous studies it was demonstrated that in TIDM, ED develops in the early stages of the disease and persists despite glycemic control is achieved (20). Since TIDM is caused by pure insulin deficiency, con- tribution of hyperglycemia to the vascular damage can be observed more clearly in the absence of conflicting comorbid conditions such as HT, hyperlipidemia, obesity and metabolic syndrome.

Flow mediated dilation is a non invasive method used for evaluation of endothelial functions mediated by prostaglandins, nitric oxide and endothelium dependent hiperpolarising factor (21). In our study FMD was found significantly lower in diabetic groups compared to controls regardless of UAE. FMD was not correlated with $\mathrm{HbAlc}$, disease duration or degree of albumin excretion but was negatively correlated with 
age. In the literature there are reports that evaluated FMD in patients with TIDM. In the study of Jin and cols., forty-eight patients with TIDM and twenty two healthy individuals were compared in regard to FMD and intima carotid thickness. FMD was found significantly lower in diabetics, supporting our results (22). Lekakis and cols. demonstrated that basal brachial artery diameters were different but FMD measurements were similar in diabetic patients with or without microalbuminuria whereas there was no correlation between FMD and HbAlc (23). In our study FMD was higher even in patients without any evidence of retinopathy or nephropathy suggesting that endothelial dysfunction occurs way before clinically overt arterial damage develops. This hypothesis is supported by Sibal and cols. who demonstrated that FMD was reduced by $\% 45$ in patients with TIDM without any complications compared to controls (24).

Endothelin-1 is a marker of ED and plays role in angiogenesis, fibrosis, inflammation and acts as a vasopressor agent causing HT (25). In our study there wasn't any significant difference in sETl level between the diabetic subgroups and the control group. There is scarce data in the literature about the level of ETl in TIDM patients compared to controls and the correlation between ET-1 and UAE rate. Most of the studies were conducted with T2DM patients and there are conflicting results suggesting that ET-1 level is not changed, increased or even decreased in diabetics (26-28). Those contradictory results might be arising from the heterogeneous group of patients in different study populations in regard to duration of diabetes or degree of glycemic control. It is also suggested that ET-1 is a peptide that functions in autocrine or paracrine ways and serum level does not directly reflect the functional amount (27). In our study ET-1 is not correlated with presence or degree of increased UAE. However in a previous study, it was demonstrated that ET-1 blockage improves diabetic nephropathy or slows the progression in mice regardless of the changes in blood pressure (29).

Levels of ICAM-1 and sVCAM-1 that are markers of inflammation were also evaluated in the present study. sICAM-1 was significantly higher in diabetics compared to control subjects but there wasn't any difference between diabetic subgroups. In the study of Sibal and cols., sICAMl was significantly higher in TIDM patients compared to controls supporting our results (24). Bruno and cols. also compared 49 T2DM patients with 15 controls and found that SICAM-1 was higher in the diabetic group especially in ones with microalbuminuria (30). In another report by Astrup and cols., 199 patients with normoalbuminuria and $192 \mathrm{pa}-$ tients with microalbuminuria were compared with each other and no significant difference was detected regarding to sICAM-1 level. In that study patients were followed for 10 years and data of the patients was reevaluated. sICAM-1 was found to be positively correlated with overall mortality (4).

In the present study, serum sVCAMl level was significantly higher in the diabetic subgroups compared to the control group. In TIDM patients with increased UAE, sVCAM-1 was significantly higher than the ones with normal UAE. A positive correlation was detected between SVCAM-1 and degree of UAE. There are reports in the literature demonstrated that serum sVCAM-1 is increased in patients with T2DM $(31,32)$. However Bannan and cols. compared TIDM with their first degree relatives and healthy subjects and they didn't detect any significant difference in regard to VCAMl between the groups (33). Our study results are supporting the report by Schmidt and cols. and Clausen and cols. who found that VCAMl was higher in diabetic patients with microalbuminuria compared to normoalbuminuric patients and control subjects $(34,35)$.

In our study there wasn't any correlation between serum ICAM-1, VCAMl and HbAlc, duration of diabetes. Muni and cols. reported the results of follow up data of DCCT trial and similar to our study they didn't detect any significant correlation between inflammatory biomarkers (c-reactive protein, ICAM-1 and VCAM-1) and $\mathrm{HbAlc}$, age and duration of diabetes (36).

There are limitations of our study. First of all the last $\mathrm{HbAlc}$ value of the patients were considered while evaluating the correlation between glycemic control and $\mathrm{ED}$. HbAlc reflects the glucose levels of last three months and relation of ED and glycemic control is complex and requires a time period. If we consider that ED begins in the early phase of the disease, it would be more appropriate to take mean of several HbAlc measurements since the diagnosis of DM. Another limiting factor is measuring FMD once for each patient. Acute hyperglycemia or acute hypoglycemia can affect FMD measurements so plasma glucose levels at the time of the measurement should have been verified $(37,38)$.

Despite the limitations, our study is the first including FMD, sET-1, sICAM-1, sVCAM-1 measurements and comparing them in subgroups of TIDM with or 
without microalbuminuria. Since serum VCAM-1 level was significantly higher in microalbuminuric patients, we assume that it may be used as a predictive marker for risk stratification of nephropathy development and progression. We also think that medical agents targeting that proinflammatory molecules can be used in the primary prevention of diabetic nephropathy and cardiovascular disease in the future.

Funding: this research received no specific grant from any funding agency in the public, commercial, or not-for-profit sectors.

Disclosure: no potential conflict of interest relevant to this article was reported.

\section{REFERENCES}

1. Heerspink HJ, de Zeeuw D. The kidney in diabetes therapy. Rev Diabet Stud. 2011;8:392-402.

2. Gerstein HC, Mann JF, Yi Q, Zinman B, Dinneen SF, Hoogwerf $B$, et al. Albuminuria and risk of cardiovascular events, death, and heart failure in diabetic and nondiabetic individuals. JAMA. $2001 ; 286(4): 421-6$.

3. Krempf M. [Specifics of diabetic nephropathy, viewpoint in diabetology: trends of diabetic nephropathy in diabetes]. NephrolTher. 2006;2 Suppl 3:S190-2.

4. Astrup AS, Tarnow L, Pietraszek L, Schalkwijk CG, Stehouwer $\mathrm{CD}$, Parving $\mathrm{HH}$, et al. Markers of endothelial dysfunction and inflammation in type 1 diabetic patients with or without diabetic nephropathy followed for 10 years: association with mortality and decline of glomerular filtration rate. Diabetes Care. 2008;31(6):1170-6.

5. Widlansky ME, Gokce N, Keaney JF Jr, Vita JA. The clinical implications of endothelial dysfunction. J Am Coll Cardiol. 2003;42(7):1149-60.

6. Vita JA, Keaney JF Jr. Endothelial function: a barometer for cardiovascular risk? Circulation. 2002;106:640-2.

7. Kalani M. The importance of endothelin 1 for microvascular dysfunction in diabetes. Vasc Health Risk Manag. 2008;4:1061-8.

8. Collier A, Leach JP, McLellan A, Jardine A, Morton JJ, Small M. Plasma endothelinlike immunoreactivity levels in IDDM patients with microalbuminuria. Diabetes Care. 1992;15(8):1038-40.

9. HaakT, Jungmann E, Felber A, Hillmann U, Usadel KH. Increased plasma levels of endothelin in diabetic patients with hypertension. Am J Hypertens. 1992;5:161-6.

10. Kawamura M, Ohgawara H, Naruse M, Suzudi N, Iwasaki N, Naruse $\mathrm{K}$, et al. Increased plasma endothelin in NIDDM patients with retinopathy. Diabetes Care. 1992;15:1396-7.

11. HuoY, Ley K. Adhesion molecules and atherogenesis. Acta Physiol Scand. 2001;173(1):35-43.

12. Sahakyan K, Klein BE, Lee KE, Tsai MY, Klein R. Inflammatory and endothelial dysfunction markers and proteinuria in persons with type 1 diabetes mellitus. Eur J Endocrinol. 2010;162(6):1101-5.

13. Matsumoto K, Sera Y, Ueki Y, Inukai G, Niiro E, Miyake S. Comparison of serum concentrations of soluble adhesion molecules in diabetic microangiopathy and macroangiopathy. Diabet Med. 2002;19(10):822-6.

14. McLeod DS, Lefer DJ, Merges J, Lutty GA. Enhanced expression of intracellular adhesion molecule-1 and P-selectin in the diabetic human retina and choroid. Am J Pathol. 1995;147:642-53.
15. Miyamoto K, Khosrof S, Bursell SE, Rohan R, Murata T, Clermont AC, et al. Prevention of leukostasis and vascular leakage in streptozotocin-induced diabetic retinopathy via intercellular adhesion molecule-1 inhibition. Proc Natl Acad Sci U S A. 1999;96(19):10836-41.

16. Taslipinar A, Yaman H, Yilmaz MI, Demirbas S, Saglam M, Taslipinar MY, et al. The relationship between inflammation, endothelial dysfunction and proteinuria in patients with diabetic nephropathy. Scand J Clin Lab Invest. 2011;71(7):606-12.

17. Corretti MC, Anderson TJ, Benjamin EJ, Celermajer D, Charbonneau F, Creager MA, et al.; International Brachial Artery Reactivity Task Force. Guidelines for the ultrasound assessment of endothelial-dependent flow-mediated vasodilation of the brachial artery: a report of the International Brachial Artery Reactivity Task Force. J Am Coll Cardiol. 2002;39(2):257-65.

18. Phillips SA, Das E, Wang J, Pritchard K, Gutterman DD. Resistance and aerobic exercise protects against acute endothelial impairment induced by a single exposure to hypertension during exertion. J Appl Physiol (1985). 2011;110(4):1013-20.

19. Sahakyan K, Klein BE, Lee KE, Tsai MY, Klein R. Inflammatory and endothelial dysfunction markers and proteinuria in persons with type 1 diabetes mellitus. Eur J Endocrinol. 2010;162(6):1101-5.

20. Ceriello A, Kumar S, Piconi L, Esposito K, Giugliano D. Simultaneous control of hyperglycemia and oxidative stress normalizes endothelial function in type 1 diabetes. Diabetes Care. 2007;30(3):649-54.

21. Creager M, Luscher TF, Cosentino F, Beckman JC. Diabetes and vascular disease: pathophysiology, clinical consequences and medical therapy: part 1. Circulation. 2003;108:1527-32.

22. Jin SM, Noh Cl, Yang SW, Bae EJ, Shin $\mathrm{CH}$, Chung HR, et al. Endothelial dysfunction and microvascular complications in type 1 diabetes mellitus. J Korean Med Sci. 2008;23(1):77-82.

23. Lekakis J, Papamichael C, Anastasiou H, Alevizaki M, Desses N, Souvatzoglou A, et al. Endothelial dysfunction of conduit arteries in insulin-dependent diabetes mellitus without microalbuminuria. Cardiovasc Res. 1997;34(1):164-8.

24. Sibal L, Aldibbiat A, Agarwal SC, Mitchell G, Oates C, Razvi S, et al. Circulating endothelial progenitor cells, endothelial function, carotid intima-media thickness and circulating markers of endothelial dysfunction in people with type 1 diabetes without macrovascular disease or microalbuminuria. Diabetologia. 2009;52(8):1464-73.

25. Al Lafi SM, Artinian SB, Boutary SS, Zwainy NS, Bitar KM, Bikhazi AB. Aliskiren, exendin-4, and insulin: their impact on endothelin receptor subtype(s) regulation/binding in type 1 diabetic rat hearts. Can J Physiol Pharmacol. 2013;91(10):830-8.

26. Takahashi K, Ghatei MA, Lam HC, O'Halloran DJ, Bloom SR. Elevated plasma endothelin in patients with diabetes mellitus. Diabetologia. 1990;33:306-10.

27. Candido R, Allen TJ. Haemodynamics in microvascular complications in type 1 diabetes. Diabetes Metab Res Rev. 2002;18(4):286304.

28. Hopfner RL, Gopalakrishnan V. Endothelin: emerging role in diabetic vascular complications. Diabetologia. 1999;42:1383-94.

29. Nakamura T, Ebihara I, Fukui M, Tomino Y, Koide H. Effect of a specific endothelin receptor $A$ antagonist on mRNA levels for extracellular matrix components and growth factors in diabetic glomeruli. Diabetes. 1995;44(8):895-9.

30. Bruno $\mathrm{CM}$, Valenti M, Bertino G, Ardiri A, Bruno F, Cunsolo $M$, et al. Plasma ICAM-1 and VCAM-1 levels in type 2 diabetic patients with and without microalbuminuria. Minerva Med. 2008;99(1):1-5.

31. Otsuki M, Hashimoto K, Morimoto $Y$, Kishimoto T, Kasayama S. Circulating vascular cell adhesion molecule-1 (VCAM-1) in atherosclerotic NIDDM patients. Diabetes. 1997;46:2096-101. 
32. Albertini JP, Valensi P, Lormeau B, Aurousseau MH, Ferriere F, Attali JR, et al. Elevated concentrations of soluble E-selectin and vascular cell adhesion molecule-1 in NIDDM. Effect of intensive insulin treatment. Diabetes Care. 1998;21(6):1008-13.

33. Bannan S, Mansfield MW, Grant PJ. Soluble vascular cell adhesion molecule-1 and E-selectin levels in relation to vascular risk factors and to Eselectin genotype in the first degree relatives of NIDDM patients and in NIDDM patients. Diabetologica. 1998;41:460-6.

34. Schmidt AM, Crandall J, Hori O, Cao R, Lakatta E. Elevated plasma levels of vascular cell adhesion molecule-1 (VCAM-1) in diabetic patients with microalbuminuria: a marker of vascular dysfunction and progressive vascular disease. Br J Haematol. 1996;92(3):747-50.

35. Clausen $P$, Jacobsen $P$, Rossing $K$, Jensen JS, Parving HH, FeldtRasmussen B. Plasma concentrations of VCAM-1 and ICAM-1 are elevated in patients with Type 1 diabetes mellitus with microalbuminuria and overt nephropathy. Diabet Med. 2000;17(9):644-9.

36. Muni RH, Kohly RP, Lee EQ, Manson JE, Semba RD, Schaumberg DA. Prospective study of inflammatory biomarkers and risk of diabetic retinopathy in the diabetes control and complications trial. JAMA Ophthalmol. 2013;131(4):514-21.

37. Har R, Lai V, Cherney D. Glucagon-like peptide 1 reduces endothelial dysfunction, inflammation, and oxidative stress induced by both hyperglycemia and hypoglycemia in type 1 diabetes. The effect of sex on endothelial function responses to clamped hyperglycemia in type 1 diabetes. Hypertens Res. 2013 Oct 3. Epub ahead of print

38. Ceriello A, Novials A, Ortega E, Canivell S, La Sala L, Pujadas G, et al. Glucagon-like peptide 1 reduces endothelial dysfunction, inflammation, and oxidative stress induced by both hyperglycemia and hypoglycemia in type 1 diabetes. Diabetes Care. 2013;36(8):2346-50. 